\title{
Factores que influyen en la prevención del cáncer de cuello uterino en la comunidad quilombolas
}

\author{
Factors that influence the prevention of cervical \\ cancer in the quilombo

\section{Fatores que influenciam na prevenção do câncer do colo do útero na comunidade quilombola}

Kamilla Santana Jacintho ${ }^{1}$, Kariane Omena Ramos Cavalcante², Jovânia Marques de Oliveira e Silva ${ }^{3}$, Amuzza Aylla Pereira dos Santos ${ }^{4}$

${ }^{1}$ Enfermeira, graduada pela Universidade Federal de Alagoas, UFAL, Unidade básica de Saúde Maceió

2 Enfermeira, graduada pela Universidade Federal de Alagoas, UFAL, Unidade básica de Saúde Maceió

3 Enfermeira, graduada pela Universidade Federal de Alagoas, UFAL, Doutora em Enfermagem, Docente da Escola de Enfermagem e Farmácia da Universidade Federal de Alagoas (ESENFAR/UFAL)

4 Enfermeira, graduada pela Universidade Federal de Pernambuco, UFPE, Doutora em Ciências da Saúde da Universidade Federal de Alagoas (UFAL) e Docente da Escola de Enfermagem e Farmácia da Universidade Federal de Alagoas

(ESENFAR/UFAL).

Cómo citar este artículo en edición digital: Jacintho, K.S., Cavalcante, K.O.R., Silva, J.M.O., Santos, A.A.P.

(2018). Factores que influyen en la prevención del cáncer de cuello uterino en la comunidad quilombolas.

Cultura de los Cuidados (Edición digital), 22(50). Recuperado de http://dx.doi.org/10.14198/cuid.2018.50.14

Corresspondencia: Amuzza Aylla Pereira dos Santos. Av. Lourival Melo Mota, s/n Campus A.C. Simões - BR 104 - Norte Km 97. Tabuleiro dos Martins. 57072970 - Maceió, AL - Brasil.

Correo electrónico: amuzzasantos@bol.com.br Recibido: 29/06/2017; Aceptado: 19/10/2017

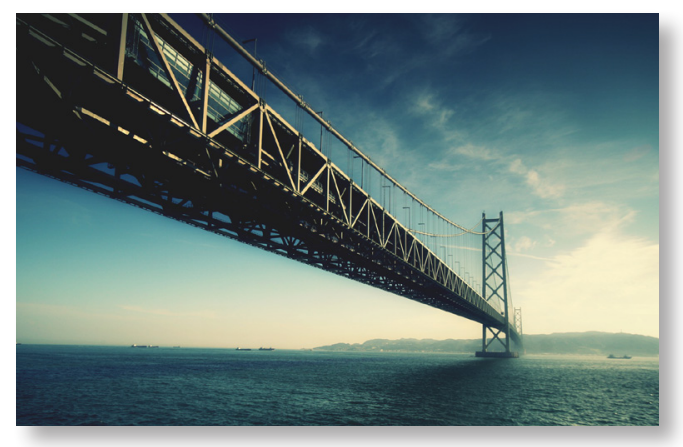

ABSTRACT

Objective: To identify factors that influence the prevention of cervical cancer in quilombola women.

Methodo: It is an ethnographic study, the study population was composed of women attending the Basic Health Unit of this community. For the data collection were used: form, observation and health education workshops. The analysis consisted of reading and re-read- ing the interviews, systematizing them, classifying the units of significance and then categorizing them.

Results: The non-adherence of the women was higher in black, elderly and low schooling. The shame and fear of exposure were other points, which hinder the performance of the preventive examination.

Conclusion: It is important to emphasize that health education is a factor that can contribute to these women know more about the preventive exam and can acquire information, clarify doubts and prevent cervical cancer.

Keywords: nursing, Prenatal Care, Women's Health, Early Detection of Cancer.

\section{RESUMEN}

Objetivo: Identificar los factores que influyen en la prevención del cáncer de cuello uterino en las mujeres quilombo. 
Método: Se trata de un estudio etnográfico, la población de estudio estaba compuesta por mujeres que acudieron a la Unidad Básica de Salud de esta comunidad. Para la recolección de datos se utilizaron: talleres de forma, de observación y de educación sanitaria. El análisis consistió en la lectura y relectura de entrevistas, sistematización de ellos, clasificación de las unidades de significación y luego categorización de los mismos.

Resultados: La falta de adherencia de las mujeres fue mayor en mujeres negras edad escolar. La vergüenza y el miedo a la exposición fueron otros puntos que dificultan la realización del cribado.

Conclusión: Es importante tener en cuenta que la educación sanitaria es un factor que puede contribuir a que estas mujeres estén más informadas acerca de la prueba de prevención también para adquirir información, aclarar dudas y prevenir el cáncer del cuello del útero.

Palabras-clave: Enfermería, Cuidado Prenatal; Salud de la Mujer, detección temprana del cáncer.

\section{RESUMO}

Objetivo: Identificar os fatores que influenciam na prevenção do câncer cérvico-uterino em mulheres quilombola.

Método: É um estudo etnográfico, a população de estudo foi composta por mulheres que frequentam a Unidade Básica de Saúde desta comunidade. Para a coleta de dados foram utilizados: formulário, observação e oficinas de educação em saúde. A análise consistiu na leitura e releitura das entrevistas, sistematização das mesmas, classificação das unidades de significância e em seguida sua categorização.

Resultados: A não adesão das mulheres foi maior em negras, de idade avançada e baixa escolaridade. A vergonha e o medo da expo-

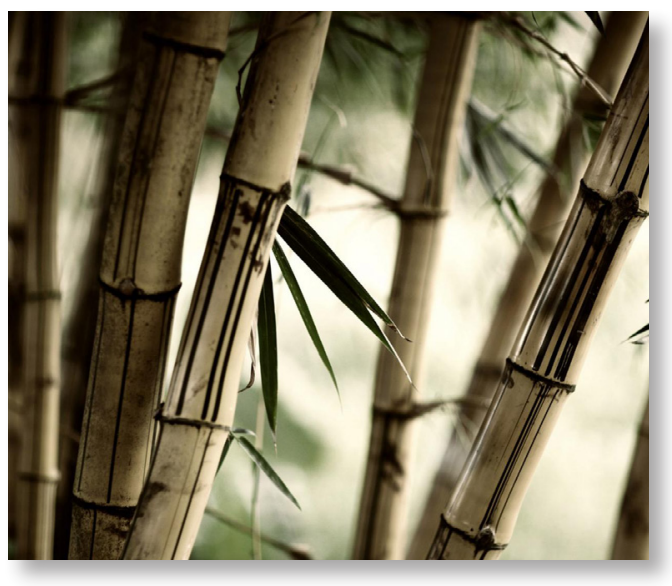

sição foram outros pontos, o que prejudicam a realização do exame preventivo.

Conclusão: É importante frisar que a educação em saúde é fator de que pode contribuir para que estas mulheres conheçam mais sobre o exame preventivo e possa adquirir informações, esclarecer dúvidas e se prevenirem contra o câncer de colo do útero.

Palavras-chave: Enfermagem, Cuidado Pré-natal; Saúde da Mulher, Detecção Precoce de Câncer.

\section{INTRODUÇAO}

O câncer do colo do útero, também chamado de cervical, demora muitos anos para se desenvolver. A detecção precoce em mulheres assintomáticas (rastreamento), por meio do exame citopatológico (Papanicolau), permite a detecção das lesões precursoras e da doença em estágios iniciais, antes mesmo do aparecimento dos sintomas. A principal alteração que pode levar a esse tipo de câncer é a infecção pelo papilomavírus humano, o HPV, com alguns subtipos de alto risco e relacionados a tumores malignos (Instituto Nacional do Câncer, 2014).

Segundo dados do Instituto Nacional do Câncer são aproximadamente 500 mil casos 
novos por ano no mundo, e aproximadamente 5.430 mulheres morrem por ano, sendo o risco estimado de 18 casos a cada 100 mil mulheres. É o terceiro tumor mais frequente na população feminina, depois do câncer de mama e do colorretal, e apresenta um dos mais altos potenciais de prevenção e cura, chegando a perto de $100 \%$, quando diagnosticado precocemente e podendo ser tratado em nível ambulatorial em cerca de $80 \%$ dos casos. A faixa etária prioritária para a detecção precoce do câncer do colo do útero é dos 35 aos 49 anos de idade, período que corresponde ao pico de incidência das lesões precursoras e antecede o agendamento de exames citológicos para prevenção e detecção do câncer de colo de útero (Instituto Nacional do Câncer, 2016).

A avaliação do câncer de colo uterino em grupos populacionais, como por exemplo, em população quilombola, permite identificar o perfil daquelas submetidas (ou não) ao exame de Papanicolau. Esses dados são fundamentais para estimar a cobertura e o foco dos programas em andamento, definir metas a serem alcançadas e priorizar as mulheres que apresentam maior risco, o que permite um planejamento mais realístico das necessidades em saúde, potencializando o impacto das ações a serem desenvolvidas e otimiza a utilização dos recursos de saúde. (Olesen; Butterworth; Jacomb \& Tait, 2012)

A principal estratégia para o rastreamento do câncer do colo do útero é a realização periódica do exame citopatológico, mais conhecido como "Papanicolaou", que consiste na análise das células oriundas da ectocérvice e da endocérvice que são extraídas por raspagem do colo do útero. Esse exame detecta lesões precocemente e faz o diagnóstico da doença em estágios iniciais, antes mesmo do aparecimento dos sintomas (Cardelli, 2015).
Nessa perspectiva os estudos comprovam que os dados socioeconômicos indicam que no Brasil, a maioria das mulheres negras encontra-se abaixo da linha de pobreza e têm menos acesso aos serviços de saúde de boa qualidade, à atenção ginecológica, além da exposição à determinadas doenças, que vão repercutir sobremaneira na atenção obstétrica (Olesen; Butterworth; Jacomb \& Tait, 2012; Haddad, 2014).

Desse modo, considerando as desfavoráveis condições socioeconômicas das mulheres negras, no contexto das comunidades quilombolas, não é diferente da condição de desigualdade social das mulheres que vivem na comunidade quilombola Muquém na cidade de União dos Palmares - Alagoas, o presente estudo tem como objetivo identificar os fatores que influenciam na prevenção do câncer cérvico-uterino em mulheres quilombola.

\section{METÓDO}

Trata-se de um estudo qualitativo, de natureza descritiva e etnografica, versando sobre a prevenção do câncer cérvico uterino em mulheres quilombolas da comunidade Muquém $\mathrm{Al}$. O estudo é de natureza etnográfica que requer do pesquisador, uma aproximação com o sujeito. O diferencial na etnografia é que essa aproximação, somente, será efetivada a partir da convivência, o que possibilitará ao pesquisador conhecer a comunidade e o contexto do sujeito do estudo, onde desenvolve seus costumes e hábitos, onde sua cultura está enraizada (Minayo, 2012).

Inicialmente, por meio de um projeto de pesquisa, o estudo foi submetido ao Comitê de Ética em Pesquisa da Universidade Federal de Alagoas e as autorizações formais obtidas junto à comunidade. Após a aprovação da pesquisa, a aproximação das mulheres deu-se por 


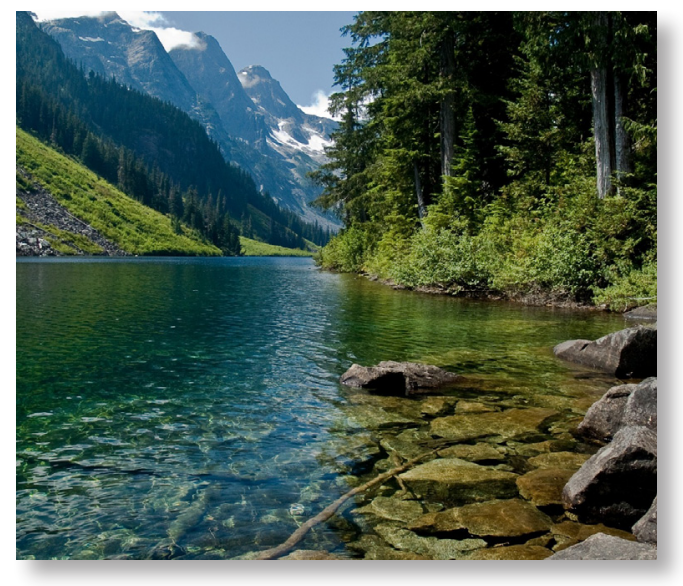

meio do esclarecimento dos aspectos relacionados ao estudo, sendo realizada a leitura e a assinatura do Termo de Consentimento Livre e Esclarecido (TCLE) por aquelas que aceitaram participar da pesquisa.

O estudo foi realizado na comunidade quilombola, denominada Muquém, localizada em União dos Palmares-Al no período de junho a dezembro/2015 e os sujeitos do estudo foram mulheres quilombolas na faixa etária de 25 a 59 anos. Este trabalho cumpriu com as normas determinadas pela Resolução 466/12 do Conselho Nacional de Saúde, que trata das diretrizes e normas regulamentadoras de pesquisa envolvendo seres humanos.

Foram entrevistadas 10 mulheres que atenderam aos critérios de inclusão: ter de 25 a 59 anos segundo a definição do Instituto Nacional do Câncer (INCA), habitar na comunidade quilombola do Muquém e aceitaram participar do referido estudo.

Para a coleta de dados, foi utilizado um formulário para a identificação e elaboração do perfil das mulheres, propiciando a observação do Lócus e seu modo de viver na comunidade. Foi utilizado ainda o diário de campo, bem como a elaboração de história de vida dos sujeitos do estudo com o fim de subsidiar a análise conferindo-lhe sustentação teórica.
Para a análise das informações seguiu as seguintes etapas: reunião em grupo para explicação do tem e objetivo, entrevistas, organização dos dados coletados, pré-análise por leitura exaustiva dos textos produzidos, e análise dos dados qualitativos com auxílio do referencial teórico para discussão foi o de Clifford Geertz (2008). Este teórico refere-se a interpretações que transformam em conhecimento científico as implicações mais gerais dessas interpretações, significados, concepções, forma, visão do mundo, identidade e cultura.

Desse modo, compreende-se a partir da Teoria de Geertz, que as formas de cada pessoa vivenciar uma experiência, apesar de ser particular está interacionada a um determinado contexto social.

\section{RESULTADOS E DISCUSSÃO}

Durante o estudo pode-se perceber que a realização do exame preventivo do câncer do colo útero, na Unidade de Saúde da Família (USF) Santa Luzia, tem uma baixa adesão o que nos levou a pressupor que fatores relacionados ao pouco conhecimento das mulheres quilombolas sobre a importância da realização do exame preventivo do câncer do colo do útero, o aparecimento da doença, onde ela está localiza e quais os seus possíveis sintomas ainda é um fator de grande relevância para que o índice desta doença continue a aumentar e oferecer riscos a sua saúde.

Geralmente, essas mulheres chegam a unidade de saúde com pouca informação do "ritual" acerca do exame e, ao se colocar diante do profissional, a mulher sente-se objeto de inspeção e associa a exposição da genitália à sexualidade; daí o sentimento de vergonha em relação a ela (sexualidade). A falta de informação em muitos casos funciona como indutor de nervosismo, ansiedade e medo, indepen- 
dentemente da idade e do nível de instrução (Mascarello, Zandonade \& Amorim, 2013).

Nesssa perspectiva pode-se perceber que em relação ao nível de conhecimento sobre a doença, podemos observar que o mesmo é muito baixo entre as mulheres da comunidade. A falta de conhecimento traz como consequência à baixa conscientização quanto ao significado, a importância do exame de Papanicolaou e o restrito acesso a assistência à saúde, devendo esse fato, em parte, ser responsável pelo número de mulheres que nunca realizaram o exame e que como consequência já descobrem a doença em estágio avançado (Eduardo et al, 2012).

As mulheres que procuram os serviços de saúde, ou seja, as que efetivamente acreditam na prevenção, pouco sabem sobre o câncer de colo uterino. E o pouco que revelam saber constitui-se em informações provenientes de fontes impessoais como a televisão e os cartazes das unidades básicas de saúde, por exemplo. O câncer do colo uterino poder ser curado quando descoberto precocemente, entretanto ainda há mulheres que desenvolvem esse tipo de câncer e morrem no Brasil, pelo fato de desconhecerem a finalidade do exame (Rangel, Lima \& Vargas, 2015).

Analisando as condições socioeconômicas das mulheres negras, no contexto das comunidades quilombolas não é diferente da condição de desigualdade social em que vivem as mulheres da comunidade quilombola Muquém. Uma pesquisa realizada em Feira de Santana, no estado da Bahia, mostrou que a não adesão ao Papanicolau foi maior em mulheres de idade mais avançada (entre 40 a 59 anos) de cor preta e de baixa escolaridade (Andrade et al, 2014).

Outro ponto importante e que chama atenção é a vergonha e/ou medo que as mulheres quilombolas têm com relação à "exposição" de sua intimidade durante consultas ginecológicas e para a realização do exame. Esses aspectos podem contribuir significativamente para que as mulheres deixem de procurar o serviço de saúde por acharem que são expostas e dentro desses aspectos sentem-se constrangidas e envergonhadas ao submeterem-se ao exame de prevenção, revelando que a vergonha se torna mais acentuada quando o profissional que o realiza é do sexo masculino. O sentimento de vergonha também pode ser apreendido pelas mulheres como uma sensação de impotência, desproteção e perda do domínio sobre o próprio corpo que a posição ginecológica proporciona (Anjos \& Ayala, 2014)

Para Rodrigues et.al. (2012) existe evidencia de dificuldades em se lidar com o corpo o que confirma o fato da vergonha e o desconforto serem motivos para as mulheres não realizarem o exame citopatológico. Esses sentimentos são comuns na realização de exames íntimos, mas devem ser confrontados quando impedem a realização de um procedimento que, podem ser decisivos para determinação precoce do câncer do colo do útero e aumento das chances de se combater esta doença.

O Papanicolau é a principal tática para perceber as lesões precursoras e fazer o diagnóstico da doença. Esse exame pode ser realizado em postos ou unidades de saúde. É de grande relevância que os serviços de saúde orientem sobre o que é e qual a importância do exame preventivo, pois sua realização periódica permite reduzir a mortalidade por esse tipo de neoplasia (Instituto nacional de Câncer, 2014)

A principal alteração que pode levar ao câncer do colo do útero é a infecção pelo papilomavírus humano, o HPV, com alguns subtipos de alto risco e relacionados a tumores malignos. De acordo com o manual do Ministério 
da Saúde para Controle dos Cânceres de Colo do Útero e da Mama, o exame preventivo, $\mathrm{Pa}$ panicolau, deve ser realizado uma vez por ano em mulheres de 25 a 60 anos (Instituto Nacional do Câncer, 2016)

O controle do câncer de colo de útero deve ser mais bem trabalhado para que seu conhecimento e a procura por exames preventivos estejam em crescente avanço, pois foi observado que as mulheres que procuraram o exame preventivo do câncer do colo do útero, em sua maioria (70\%) não têm o conhecimento sobre como realizar o exame preventivo, mesmo apresentando familiares que já tiveram essa patologia (Eduardo et al, 2012).

Nessa perspectiva a educação em saúde pode ser utilizada como uma estratégia de adesão para os cuidados com a saúde daquela comunidade e ainda a utilização de abordagens sobre os temas relacionados ao bem-estar físico, psicológico e o biológico, por meio da sala de espera, em locais dentro da comunidade. Essa estratégia constitui um fator que pôde contribuir para que a população possa esclarecer suas dúvidas e buscar a promoção da saúde (Castro, 2013).

Foi observado ainda, que na comunidade quilombola as mulheres realizavam os exames citopatológicos preventivos, mas não com a periodicidade que o Ministério da saúde solicita. O que confere com os $90 \%$ das entrevistadas já ter realizado o exame. Elas relataram que seu retorno a unidade básica de saúde para o exame preventivo foi baseado na busca ativa realizada, nas palestras informativas e nas informações adquiridas no decorrer das consultas de enfermagem e nos murais informativos presentes na unidade de saúde.

As práticas da Prevenção do Câncer do Colo do Útero (PCCU), ainda hoje, representam um importante desafio de saúde pública, pois as razões para isto devem-se aos fatores culturais, sociais, econômicos e comportamentais, bem como à própria organização dos serviços públicos de saúde. Além desses fatores, a demora das mulheres em realizarem o exame ou a ausência desse no serviço pode estar associada ao modo como a usuária percebe o exame preventivo. A percepção das mulheres quanto ao exame é influenciada pelos valores, pela cultura, pela raça, pelas experiências vividas, crenças, expectativas de vida e ideias pré-concebidas construídas ao longo da vida (Mascarello, Zandonade \& Amorim, 2013).

Vencer essas barreiras para uma melhor adesão das mulheres quilombolas ao exame preventivo significa dar atenção aos relatos $\mathrm{e}$ às experiências de quem a ele se submete, pois, a cada ano mais mulheres adoecem por falta de conhecimento sobre como prevenir o câncer de colo do útero, além do fato do profissional de saúde não está preparado para lidar com situações que colocam a mulher em situações constrangedoras. Para que essa prática possa mudar é preciso que as informações apresentadas, os profissionais de saúde, dentre eles o enfermeiro, consiga planejar e orientar os serviços de prevenção com vistas à promoção da saúde, esse é o primeiro caminho para diminuição da incidência desse tipo de câncer (Eduardo et al, 2012).

\section{CONCLUSAO}

$\mathrm{O}$ estudo revelou que fatores como a falta de orientação acerca da importância do acompanhamento ginecológico e da realização anual do exame citopatológico, bem como a ausência de sintomatologia, o medo e o constrangimento na realização do exame podem estar relacionados com a não realização periódica do exame. Nesse sentido as mulheres quilombolas sofrem com a falta de orientação 
e informações acerca da prevenção para câncer de colo, pois é uma população carente de serviços de saúde que proporcione a devida atenção que elas mereçam.

O conhecimento destes fatores, pode ajudar na construção de atividades junto a comunidade quilombola que possa planejar um cuidado efetivo a mulher no que se refere à prevenção do câncer de colo do útero, tornando importante destacar a compreensão acerca das alterações patológicas promovidas pela doença e os cuidados necessários para sua prevenção, possam ajudar na assistência de qualidade para essas mulheres.

\section{BIBLIOGRAFÍA:}

- Andrade, M. S., Almeida, M. M. G., Araujo, T. M.. \& Santos, K. O. B. (2014). Fatores associados a não adesão ao Papanicolau entre mulheres atendidas pela estratégia saúde da família, Brasília: Epidemiol. Serv. Saúde. 23(1), 111- 120

- Cardelli A. A. M., et al. (2015). Fatores relacionados a não adesão à realização do exame de Papanicolau. Rev Rene, 16(4), 532-539.

- Castro, M. B. (2013). A natureza jurídica da propriedade Quilombola. Pernambuco. Recuperado em 06 de fevereiro, 2015, de http://www.conteudojuridico.com. br/artigo,a-natureza-juridica-da-propriedade-quilombola,44136.html.

- Eduardo K. G. T., Moura E. R. F., Nogueira P. S. F., Costa C. B. J. S. Pinheiro A. K. B., \& Silva R. M. (2012). Conhecimento e mudanças de comportamento de mulheres junto a fatores de risco para câncer de colo uterino. Rev Rene, 13(5), 1045-1055.

- Fino, C.N. (2015). A etnografia enquanto método: um modo de entender as culturas (escolares) locais. Recuperado em 06 de fevereiro, 2015 de, http://www3.uma.pt/ carlosfino/publicacoes/22.pdf.

- Geertz C. (2008). A Interpretação das Culturas. São Paulo: LTC.

- Haddad C.F. (2014). Características clínico-patológicas e estadiamento ao diagnóstico de pacientes com câncer de mama em um centro de saúde do interior de Minas Gerais. Rev Bras Mastologia, 24(4),103- 108.

- Höfelmann D. A., Anjos J.C., \& Ayala A. L. (2014). Sobrevida em dez anos e fatores prognósticos em mulheres com câncer de mama em Joinville, Santa Catarina, Brasil. Cienc Saúde Colet, 19(6),1813-24.

- Instituto Nacional do Câncer. Câncer do colo do útero. Recuperado em 31 de março, 2014, de http://www2.inca. gov.br/wps/wcm/connect/tiposdecancer/site/home/ colo_utero/definidef.

- Instituto Nacional do Câncer. Brasil. Ministério da Saúde (MS). Instituto Nacional de Câncer (Inca). Diretrizes brasileiras para o rastreamento do câncer do colo do útero. Rio de Janeiro: Inca; 2016.

- Mascarello K.C., Zandonade E., \& Amorim M. H. C. (2013). Análise da sobrevida de mulheres com câncer do colo do útero atendidas em hospital de referência para oncologia no Espírito Santo, Brasil, nos anos de 2000 a 2005. Cad. Saúde Pública, 29(4), 823-831.

- Minayo M. C. (2012). Análise qualitativa: teoria, passos e fidedignidade. Ciência \& Saúde Coletiva, 17(3), 621-626.

- Olesen, S. C., Butterworth, P., Jacomb, P., \& Tait, R. J. (2012). Personal factors influence use of cervical cancer screening services: epidemiological survey and linked administrative data address the limitations of previous research. BMC Health Serv Res, 11, 75-86.

- Rangel G., Lima L. D., \& Vargas, E. P. (2015). Condicionantes do diagnóstico tardio do câncer cervical na ótica das mulheres atendidas no Inca. Saúde debate, 39(107),1065-1078.

- Rodrigues, B. C., Carneiro, A. C. M. O., Silva, T. L., Solá, A. C. N., Manzi, N. M., Schechtman, N. P., Magalhães, H. L. G. O. \& Dytz, J. L. G. (2012). Educação em saúde para a prevenção do câncer cérvico-úterino., Rev. Bras. Edc. Med, 36 (supl. 1), 149-154.

- Soares, M. B. O., \& Silva, S. R. (2010). Resultados de citologia oncótica em uma regional de saúde no período de 2001-2008. Rev. Rene. 11(Espec), 23-31. 